\title{
Complex Multivariate Montgomery Type Identity Leading to Complex Multivariate Ostrowski and Grüss Inequalities
}

\author{
George A. Anastassiou 1*
}

\section{Abstract}

We give a general complex multivariate Montgomery type identity which is a representation formula for a complex multivariate function. Using it we produce general tight complex multivariate high order Ostrowski and Grüss type inequalities. The estimates involve $L_{p}$ norms, any $1 \leq p \leq \infty$. We include also applications.

Keywords: Multivariate complex integral, Multivariate complex continuous functions, Multivariate complex anaIytic functions, Multivariate complex montgomery identity, Multivariate complex Ostrowski and Grüss inequalities. 2010 AMS: Primary 26D10, Secondery 26D15, 30A10.

\section{${ }^{1}$ Department of Mathematical Sciences University of Memphis Memphis, TN 38152, U.S.A.}

*Corresponding author: ganastss@memphis.edu

Received: 22 April 2019, Accepted: 14 June 2019, Available online: 27 June 2019

\section{Introduction}

Our motivation comes from the following results:

Theorem 1.1. (A. Ostrowski, 1938 [1]). Let $f:[a, b] \rightarrow \mathbb{R}$ be continuous on $[a, b]$ and differentiable on $(a, b)$ such that $f^{\prime}:(a, b) \rightarrow \mathbb{R}$ is bounded on $(a, b)$, i.e., $\left\|f^{\prime}\right\|_{\infty}:=\sup _{t \in(a, b)}\left|f^{\prime}(t)\right|<\infty$. Then

$$
\left|\frac{1}{b-a} \int_{a}^{b} f(t) d t-f(x)\right| \leq\left[\frac{1}{4}+\left(\frac{x-\frac{a+b}{2}}{b-a}\right)^{2}\right]\left\|f^{\prime}\right\|_{\infty}(b-a),
$$

for all $x \in[a, b]$ and the constant $\frac{1}{4}$ is the best possible.

Theorem 1.2. (G. Grüss, 1934 [2]). Let $f, g:[a, b] \rightarrow \mathbb{R}$ be Lebesgue integrable functions, and $m, M, n, N \in \mathbb{R}$ such that: $-\infty<m \leq f \leq M<\infty,-\infty<n \leq g \leq N<\infty$, a.e. on $[a, b]$. Then

$$
\left|\frac{1}{b-a} \int_{a}^{b} f(t) g(t) d t-\left(\frac{1}{b-a} \int_{a}^{b} f(t) d t\right)\left(\frac{1}{b-a} \int_{a}^{b} g(t) d t\right)\right| \leq \frac{1}{4}(M-m)(N-n),
$$

with the constant $\frac{1}{4}$ being the best possible. 
Let $f \in C^{1}([a, b])$ and the kernel $p:[a, b]^{2} \rightarrow \mathbb{R}$ be such that

$$
p(x, t):= \begin{cases}t-a, & \text { if } t \in[a, x], \\ t-b, & \text { if } t \in(x, b] .\end{cases}
$$

Then, we have the basic Montgomery integral identity [3, p. 565],

$$
f(x)=\frac{1}{b-a} \int_{a}^{b} f(t) d t+\frac{1}{b-a} \int_{a}^{b} p(x, t) f^{\prime}(t) d t, \forall x \in[a, b] .
$$

In order to describe complex extensions of Ostrowski and Grüss inequalities using the complex integral we need the following preparation.

Suppose $\gamma$ is a smooth path parametrized by $z(t), t \in[a, b]$ and $f$ is a complex function which is continuous on $\gamma$. Put $z(a)=u$ and $z(b)=w$ with $u, w \in \mathbb{C}$. We define the integral of $f$ on $\gamma_{u, w}=\gamma$ as

$$
\int_{\gamma} f(z) d z=\int_{\gamma_{u, w}} f(z) d z:=\int_{a}^{b} f(z(t)) z^{\prime}(t) d t .
$$

We observe that the actual choice of parametrization of $\gamma$ does not matter.

This definition immediately extends to paths that are piecewise smooth. Suppose $\gamma$ is parametrized by $z(t), t \in[a, b]$, which is differentiable on the intervals $[a, c]$ and $[c, b]$, then assuming that $f$ is continuous on $\gamma$ we define

$$
\int_{\gamma_{u, w}} f(z) d z:=\int_{\gamma_{u, v}} f(z) d z+\int_{\gamma_{, w}} f(z) d z
$$

where $v:=z(c)$. This can be extended for a finite number of intervals.

We also define the integral with respect to arc-length

$$
\int_{\gamma_{u, w}} f(z)|d z|:=\int_{a}^{b} f(z(t))\left|z^{\prime}(t)\right| d t
$$

and the length of the curve $\gamma$ is then

$$
l(\gamma)=\int_{\gamma_{u, w}}|d z|:=\int_{a}^{b}\left|z^{\prime}(t)\right| d t .
$$

Let $f$ and $g$ be holomorphic in $G$, an open domain and suppose $\gamma \subset G$ is a piecewise smooth path from $z(a)=u$ to $z(b)=w$. Then we have the integration by parts formula

$$
\int_{\gamma_{u, w}} f(z) g^{\prime}(z) d z=f(w) g(w)-f(u) g(u)-\int_{\gamma_{u, w}} f^{\prime}(z) g(z) d z .
$$

We recall also the triangle inequality for the complex integral, namely

$$
\left|\int_{\gamma} f(z) d z\right| \leq \int_{\gamma}|f(z)||d z| \leq\|f\|_{\gamma, \infty} l(\gamma),
$$

where $\|f\|_{\gamma, \infty}:=\sup _{z \in \gamma}|f(z)|$.

We also define the $p$-norm with $p \geq 1$ by

$$
\|f\|_{\gamma, p}:=\left(\int_{\gamma}|f(z)|^{p}|d z|\right)^{\frac{1}{p}}
$$

For $p=1$ we have

$$
\|f\|_{\gamma, 1}:=\int_{\gamma}|f(z)||d z| .
$$

If $p, q>1$ with $\frac{1}{p}+\frac{1}{q}=1$, then by Hölder's inequality we have

$$
\|f\|_{\gamma, 1} \leq[l(\gamma)]^{\frac{1}{q}}\|f\|_{\gamma, p} .
$$


First, we mention a Complex extension of Ostrowski inequality to the complex integral by providing upper bounds for the quantity

$$
\left|f(v)(w-u)-\int_{\gamma} f(z) d z\right|
$$

under the assumption that $\gamma$ is a smooth path parametrized by $z(t), t \in[a, b], u=z(a), v=z(x)$ with $x \in(a, b)$ and $w=z(b)$ while $f$ is holomorphic in $G$, an open domain and $\gamma \subset G$.

Secondly, we mention a Complex extension of Grüss inequality:

Suppose $\gamma \subset \mathbb{C}$ is a piecewise smooth path parametrized by $z(t), t \in[a, b]$ from $z(a)=u$ to $z(b)=w$ with $w \neq u$. If $f$ and $g$ are continuous on $\gamma$, we consider the complex Čebyšev functional defined by

$$
\mathscr{D}_{\gamma}(f, g):=\frac{1}{w-u} \int_{\gamma} f(z) g(z) d z-\frac{1}{w-u} \int_{\gamma} f(z) d z \frac{1}{w-u} \int_{\gamma} g(z) d z .
$$

We display upper bounds to $\left|\mathscr{D}_{\gamma}(f, g)\right|$.

We have the following results for functions of a complex variable:

Theorem 1.3. (S. Dragomir, 2019 [4]). Let $f$ be holomorhic in $G$, an open domain and suppose $\gamma \subset G$ is a smooth path from $z(a)=u$ to $z(b)=w$. If $v=z(x)$ with $x \in(a, b)$, then $\gamma_{u, w}=\gamma_{u, v} \cup \gamma_{v, w}$,

$$
\begin{aligned}
& \left|f(v)(w-u)-\int_{\gamma} f(z) d z\right| \leq\left\|f^{\prime}\right\|_{\gamma_{u, v} ; \infty} \int_{\gamma_{u, v}}|z-u||d z|+\left\|f^{\prime}\right\|_{\gamma_{v, w} ; \infty} \int_{\gamma_{u, w}}|z-w||d z| \leq \\
& {\left[\int_{\gamma_{u, v}}|z-u||d z|+\int_{\gamma_{u, w}}|z-w||d z|\right]\left\|f^{\prime}\right\|_{\gamma_{u, w} ; \infty},}
\end{aligned}
$$

and

$$
\begin{aligned}
& \left|f(v)(w-u)-\int_{\gamma} f(z) d z\right| \leq \max _{z \in \gamma_{u, v}}|z-u|\left\|f^{\prime}\right\|_{\gamma_{u, v} ; 1}+\max _{z \in \gamma_{v, w}}|z-w|\left\|f^{\prime}\right\|_{\gamma_{v, w} ; 1} \leq \\
& \max \left\{\max _{z \in \gamma_{u, v}}|z-u|, \max _{z \in \gamma_{v, w}}|z-w|\right\}\left\|f^{\prime}\right\|_{\gamma_{u, w} ; 1} .
\end{aligned}
$$

If $p, q>1$ with $\frac{1}{p}+\frac{1}{q}=1$, then

$$
\begin{aligned}
& \left|f(v)(w-u)-\int_{\gamma} f(z) d z\right| \leq\left(\int_{\gamma_{u, v}}|z-u|^{q}|d z|\right)^{\frac{1}{q}}\left\|f^{\prime}\right\|_{\gamma_{u, v} ; p}+\left(\int_{\gamma_{u, w}}|z-w|^{q}|d z|\right)^{\frac{1}{q}}\left\|f^{\prime}\right\|_{\gamma_{v, w} ; p} \leq \\
& \left(\int_{\gamma_{u, v}}|z-u|^{q}|d z|+\int_{\gamma_{u, w}}|z-w|^{q}|d z|\right)^{\frac{1}{q}}\left\|f^{\prime}\right\|_{\gamma_{u, w} ; p} \cdot
\end{aligned}
$$

Suppose $\gamma \subset \mathbb{C}$ is a piecewise smooth path parametrized by $z(t), t \in[a, b]$ from $z(a)=u$ to $z(b)=w$. Now, for $\phi, \Phi \in \mathbb{C}$ define the set of complex-valued functions

$$
\bar{\Delta}_{\gamma}(\phi, \Phi):=\left\{f: \gamma \rightarrow \mathbb{C}|| f(z)-\frac{\phi+\Phi}{2}\left|\leq \frac{1}{2}\right| \Phi-\phi \mid \text { for each } z \in \gamma\right\} .
$$

We have the following complex Grüss type inequalities:

Theorem 1.4. (S. Dragomir, 2018 [5]). Suppose $\gamma \subset \mathbb{C}$ is a piecewise smooth path parametrized by $z(t), t \in[a, b]$ from $z(a)=u$ to $z(b)=w$ with $w \neq u$. If $f$ and $g$ are continuous on $\gamma$ and there exist $\phi, \Phi, \psi, \Psi \in \mathbb{C}, \phi \neq \Phi, \psi \neq \Psi$ such that $f \in \bar{\Delta}_{\gamma}(\phi, \Phi)$ and $g \in \bar{\Delta}_{\gamma}(\psi, \Psi)$ then

$$
\left|\mathscr{D}_{\gamma}(f, g)\right| \leq \frac{1}{4}|\Phi-\phi||\Psi-\psi| \frac{l^{2}(\gamma)}{|w-u|^{2}}
$$


If the path $\gamma$ is a segment $[u, w]$ connecting two distinct points $u$ and $w$ in $\mathbb{C}$ then we write $\int_{\gamma} f(z) d z$ as $\int_{u}^{w} f(z) d z$.

If $f, g$ are continuous on $[u, w]$ and there exists $\phi, \Phi, \psi, \Psi \in \mathbb{C}, \phi \neq \Phi, \psi \neq \Psi$ such that $f \in \bar{\Delta}_{[u, w]}(\phi, \Phi)$ and $g \in \bar{\Delta}_{[u, w]}(\psi, \Psi)$ then

$$
\left|\frac{1}{w-u} \int_{u}^{w} f(z) g(z) d z-\frac{1}{w-u} \int_{u}^{w} f(z) d z \frac{1}{w-u} \int_{u}^{w} g(z) d z\right| \leq \frac{1}{4}|\Phi-\phi||\Psi-\psi| .
$$

We will use the complex Montgomery identity which follows:

Theorem 1.5. (S. Dragomir, 2018 [4]) Let $f$ be holomorphic in $G$, an open domain and suppose $\gamma \subset G$ is a smooth path from $z(a)=u$ to $z(b)=w$. If $v=z(t)$ with $t \in[a, b]$, then $\gamma_{u, w}=\gamma_{u, v} \cup \gamma_{v, w}$, and

$$
f(v)=\frac{1}{w-u}\left[\int_{\gamma} f(z) d z+\int_{\gamma_{u, v}}(z-u) f^{\prime}(z) d z+\int_{\gamma_{v, w}}(z-w) f^{\prime}(z) d z\right] .
$$

Define

$$
p(v, z):=\left\{\begin{aligned}
z-u, & \text { if } z \in \gamma_{u, v} \\
z-w, & \text { if } z \in \gamma_{v, w}
\end{aligned}\right.
$$

Thus, it holds

$$
f(v)=\frac{1}{w-u} \int_{\gamma} f(z) d z+\frac{1}{w-u} \int_{\gamma} p(v, z) f^{\prime}(z) d z,
$$

a form which we will use a lot in this article.

Representation formula (1.1) is the main inspiration to write this article.

We will use (1.1) to derive a multivariate Complex Montgomery type identity then based on it, we will produce Complex multivariate Ostrowski and Grüss type inequalities.

For the last we need:

Definition 1.6. Here we extend the notion of line (curve) integral into multivariate case. Let $\gamma_{j}, j=1, \ldots, m$, be a smooth path parametrized by $z_{j}\left(t_{j}\right), t_{j} \in\left[a_{j}, b_{j}\right]$ and $f$ is a complex valued function which is continuous on $\prod_{j=1}^{m} \gamma_{j} \subseteq \mathbb{C}^{m}$. Put $z_{j}\left(a_{j}\right)=u_{j}$ and $z_{j}\left(b_{j}\right)=w_{j}$, with $u_{j}, w_{j} \in \mathbb{C}, j=1, \ldots, m$.

We define the complex multivariate integral of $f$ on $\prod_{j=1}^{m} \gamma_{j}:=\prod_{j=1}^{m} \gamma_{u_{j}, w_{j}}$ as

$$
\begin{aligned}
& \int_{\gamma_{1}} \ldots \int_{\gamma_{m}} f\left(z_{1}, \ldots, z_{m}\right) d z_{1} \ldots d z_{m}:=\int_{\prod_{j=1}^{m} \gamma_{j}} f\left(z_{1}, \ldots, z_{m}\right) d z_{1} \ldots d z_{m}:= \\
& \int_{\gamma_{u_{1}, w_{1}}} \ldots \int_{\gamma_{u_{m}, w_{m}}} f\left(z_{1}, \ldots, z_{m}\right) d z_{1} \ldots d z_{m}:=\int_{\prod_{j=1}^{m} \gamma_{u_{j}, w_{j}} f\left(z_{1}, \ldots, z_{m}\right) d z_{1} \ldots d z_{m}:=} \\
& \int_{a_{1}}^{b_{1}} \int_{a_{2}}^{b_{2}} \ldots \int_{a_{m}}^{b_{m}} f\left(z_{1}\left(t_{1}\right), \ldots, z_{m}\left(t_{m}\right)\right) \prod_{j=1}^{m} z_{j}^{\prime}\left(t_{j}\right) d t_{1} \ldots d t_{m} .
\end{aligned}
$$

We make

Remark 1.7. Clearly here $z_{j} \in C^{1}\left(\left[a_{j}, b_{j}\right], \mathbb{C}\right), j=1, \ldots, m$. The integrand in (1.2) is a continuous complex valued function over $\prod_{j=1}^{m}\left[a_{j}, b_{j}\right]$. Therefore $\left|f\left(z_{1}\left(t_{1}\right), \ldots, z_{m}\left(t_{m}\right)\right)\right| \prod_{j=1}^{m} z_{j}^{\prime}\left(t_{j}\right)$ is also continuous but from $\prod_{j=1}^{m}\left[a_{j}, b_{j}\right]$ into $\mathbb{R}$, hence it is bounded. Consequently it holds

$$
\int_{j=1}^{m}\left[a_{j}, b_{j}\right]\left|f\left(z_{1}\left(t_{1}\right), \ldots, z_{m}\left(t_{m}\right)\right)\right| \prod_{j=1}^{m}\left|z_{j}^{\prime}\left(t_{j}\right)\right| \prod_{j=1}^{m} d t_{j}<+\infty .
$$


Therefore, by Fubini's theorem, the order integration in (1.2) is immaterial.

Clearly it holds

$$
\left|\int_{a_{1}}^{b_{1}} \ldots \int_{a_{m}}^{b_{m}} f\left(z_{1}\left(t_{1}\right), \ldots, z_{m}\left(t_{m}\right)\right) \prod_{j=1}^{m} z_{j}^{\prime}\left(t_{j}\right) d t_{1} \ldots d t_{m}\right| \leq \int_{a_{1}}^{b_{1}} \ldots \int_{a_{m}}^{b_{m}}\left|f\left(z_{1}\left(t_{1}\right), \ldots, z_{m}\left(t_{m}\right)\right)\right| \prod_{j=1}^{m}\left|z_{j}^{\prime}\left(t_{j}\right)\right| d t_{1} \ldots d t_{m} .
$$

We also define the integral with respect to arc-lengths

$$
\int_{j=1}^{m} \gamma_{u_{j}, w_{j}} f\left(z_{1}, \ldots, z_{m}\right)\left|d z_{1}\right|\left|d z_{2}\right| \ldots\left|d z_{m}\right|:=\int_{\prod_{j=1}^{m}\left[a_{j}, b_{j}\right]} f\left(z_{1}\left(t_{1}\right), \ldots, z_{m}\left(t_{m}\right)\right) \prod_{j=1}^{m}\left|z_{j}^{\prime}\left(t_{j}\right)\right| d t_{1} \ldots d t_{m} .
$$

It holds (by (1.3), (1.4))

$$
\left|\int_{\prod_{j=1}^{m} \gamma_{j}} f\left(z_{1}, \ldots, z_{m}\right) d z_{1} \ldots d z_{m}\right| \leq \int_{\prod_{j=1}^{m} \gamma_{u_{j}, w_{j}}}\left|f\left(z_{1}, \ldots, z_{m}\right)\right|\left|d z_{1}\right|\left|d z_{2}\right| \ldots\left|d z_{m}\right| \leq\|f\| \prod_{j=1}^{m} \gamma_{j} \prod_{j=1}^{m} l\left(\gamma_{j}\right)
$$

where

$$
\|f\| \prod_{j=1}^{m} \gamma_{j}:=\sup _{\left(z_{1}, \ldots, z_{m}\right) \in \prod_{j=1}^{m} \gamma_{j}}\left|f\left(z_{1}, \ldots, z_{m}\right)\right|,
$$

and

$$
l\left(\gamma_{j}\right)=\int_{\gamma_{u_{j}}, w_{j}}\left|d z_{j}\right|=\int_{a_{j}}^{b_{j}}\left|z_{j}^{\prime}\left(t_{j}\right)\right| d t_{j}, \quad j=1, \ldots, m .
$$

We also define the $p$-norm with $p \geq 1$ by

$$
\|f\| \prod_{j=1}^{m} \gamma_{j}, p=\left(\int_{\prod_{j=1}^{m} \gamma_{j}}\left|f\left(z_{1}, \ldots, z_{m}\right)\right|^{p}\left|d z_{1}\right|\left|d z_{2}\right| \ldots\left|d z_{m}\right|\right)^{\frac{1}{p}} .
$$

For $p=1$ we have

$$
\|f\|_{j=1}^{m} \gamma_{j}, 1=\int_{\prod_{j=1}^{m} \gamma_{j}}\left|f\left(z_{1}, \ldots, z_{m}\right)\right|\left|d z_{1}\right|\left|d z_{2}\right| \ldots\left|d z_{m}\right| .
$$

If $p, q>1$ with $\frac{1}{p}+\frac{1}{q}=1$, then by Hölder's inequality we have

$$
\|f\|_{\prod_{j=1}^{m} \gamma_{j}, 1} \leq\left(\prod_{j=1}^{m} l\left(\gamma_{j}\right)\right)^{\frac{1}{q}}\|f\|_{\prod_{j=1}^{m} \gamma_{j}, p} .
$$

\section{Main results}

We start by presenting a complex trivariate Montgomery type representation identity of complex functions:

Theorem 2.1. Let $f: \prod_{j=1}^{3} D_{j} \subseteq \mathbb{C}^{3} \rightarrow \mathbb{C}$ be a continuous function that is analytic per coordinate on the domain $D_{j}$, $j=1,2,3$, and $x=\left(x_{1}, x_{2}, x_{3}\right) \in \prod_{j=1}^{3} D_{j}$. For $j=1,2,3$, suppose $\gamma_{j} \subset D_{j}$ is a smooth path parametrized by $z_{j}\left(t_{j}\right), t_{j} \in\left[a_{j}, b_{j}\right]$ with $z_{j}\left(a_{j}\right)=u_{j}, z_{j}\left(t_{j}\right)=x_{j}$ and $z_{j}\left(b_{j}\right)=w_{j}$, where $u_{j}, w_{j} \in D_{j}, u_{j} \neq w_{j}$. Assume also that all partial derivatives of $f$ up to order three are continuous functions on $\prod_{j=1}^{3} D_{j}$. 
Here we define the kernels for $i=1,2,3, p_{i}: \gamma_{i}^{2} \rightarrow \mathbb{C}$

$$
p_{i}\left(x_{i}, s_{i}\right):=\left\{\begin{array}{c}
s_{i}-u_{i}, \text { if } s_{i} \in \gamma_{u_{i}, x_{i}}, \\
s_{i}-w_{i}, \text { if } s_{i} \in \gamma_{x_{i}, w_{i}} .
\end{array}\right.
$$

Then

$$
\begin{gathered}
f\left(x_{1}, x_{2}, x_{3}\right)=\frac{1}{\prod_{i=1}^{3}\left(w_{i}-u_{i}\right)}\left\{\int_{\gamma_{1}} \int_{\gamma_{2}} \int_{\gamma_{3}} f\left(s_{1}, s_{2}, s_{3}\right) d s_{3} d s_{2} d s_{1}+\sum_{j=1}^{3}\left(\int_{\gamma_{1}} \int_{\gamma_{2}} \int_{\gamma_{3}} p_{j}\left(x_{j}, s_{j}\right) \frac{\partial f\left(s_{1}, s_{2}, s_{3}\right)}{\partial s_{j}} d s_{3} d s_{2} d s_{1}\right)\right. \\
\left.+\sum_{\substack{l=1 \\
j<k}}^{3}\left(\int_{\gamma_{1}} \int_{\gamma_{2}} \int_{\gamma_{3}} p_{j}\left(x_{j}, s_{j}\right) p_{k}\left(x_{k}, s_{k}\right) \frac{\partial^{2} f\left(s_{1}, s_{2}, s_{3}\right)}{\partial s_{k} \partial s_{j}} d s_{3} d s_{2} d s_{1}\right)(l)+\int_{\gamma_{1}} \int_{\gamma_{2}} \int_{\gamma_{3}}\left(\prod_{i=1}^{3} p_{i}\left(x_{i}, s_{i}\right)\right) \frac{\partial^{3} f\left(s_{1}, s_{2}, s_{3}\right)}{\partial s_{3} \partial s_{2} \partial s_{1}} d s_{3} d s_{2} d s_{1}\right\} .
\end{gathered}
$$

Above l counts $(j, k): j<k ; j, k \in\{1,2,3\}$.

Proof. Here we apply (1.1) repeatedly.

First we see that

$$
f\left(x_{1}, x_{2}, x_{3}\right)=A_{0}+B_{0},
$$

where

$$
A_{0}:=\frac{1}{w_{1}-u_{1}} \int_{\gamma_{1}} f\left(s_{1}, x_{2}, x_{3}\right) d s_{1},
$$

and

$$
B_{0}:=\frac{1}{w_{1}-u_{1}} \int_{\gamma_{1}} p_{1}\left(x_{1}, s_{1}\right) \frac{\partial f\left(s_{1}, x_{2}, x_{3}\right)}{\partial s_{1}} d s_{1} .
$$

Furthermore we have

$$
f\left(s_{1}, x_{2}, x_{3}\right)=A_{1}+B_{1},
$$

where

$$
A_{1}:=\frac{1}{w_{2}-u_{2}} \int_{\gamma_{2}} f\left(s_{1}, s_{2}, x_{3}\right) d s_{2},
$$

and

$$
B_{1}:=\frac{1}{w_{2}-u_{2}} \int_{\gamma_{2}} p_{2}\left(x_{2}, s_{2}\right) \frac{\partial f\left(s_{1}, s_{2}, x_{3}\right)}{\partial s_{2}} d s_{2} .
$$

Also we find that

$$
\begin{aligned}
& f\left(s_{1}, s_{2}, x_{3}\right)=\frac{1}{w_{3}-u_{3}} \int_{\gamma_{3}} f\left(s_{1}, s_{2}, s_{3}\right) d s_{3}+ \\
& \frac{1}{w_{3}-u_{3}} \int_{\gamma_{3}} p_{3}\left(x_{3}, s_{3}\right) \frac{\partial f\left(s_{1}, s_{2}, s_{3}\right)}{\partial s_{3}} d s_{3} .
\end{aligned}
$$

Next we put things together, and we derive

$$
A_{1}=\frac{1}{\left(w_{2}-u_{2}\right)\left(w_{3}-u_{3}\right)} \int_{\gamma_{2}} \int_{\gamma_{3}} f\left(s_{1}, s_{2}, s_{3}\right) d s_{3} d s_{2}+\frac{1}{\left(w_{2}-u_{2}\right)\left(w_{3}-u_{3}\right)} \int_{\gamma_{2}} \int_{\gamma_{3}} p_{3}\left(x_{3}, s_{3}\right) \frac{\partial f\left(s_{1}, s_{2}, s_{3}\right)}{\partial s_{3}} d s_{3} d s_{2} .
$$


And we get

$$
\begin{aligned}
& A_{0}=\frac{1}{\prod_{i=1}^{3}\left(w_{i}-u_{i}\right)} \int_{\gamma_{1}} \int_{\gamma_{2}} \int_{\gamma_{3}} f\left(s_{1}, s_{2}, s_{3}\right) d s_{3} d s_{2} d s_{1}+\frac{1}{\prod_{i=1}^{3}\left(w_{i}-u_{i}\right)} \int_{\gamma_{1}} \int_{\gamma_{2}} \int_{\gamma_{3}} p_{3}\left(x_{3}, s_{3}\right) \frac{\partial f\left(s_{1}, s_{2}, s_{3}\right)}{\partial s_{3}} d s_{3} d s_{2} d s_{1} \\
& +\frac{1}{\left(w_{1}-u_{1}\right)\left(w_{2}-u_{2}\right)} \int_{\gamma_{1}} \int_{\gamma_{2}} p_{2}\left(x_{2}, s_{2}\right) \frac{\partial f\left(s_{1}, s_{2}, x_{3}\right)}{\partial s_{2}} d s_{2} d s_{1} .
\end{aligned}
$$

Also we obtain

$$
\frac{\partial f\left(s_{1}, s_{2}, x_{3}\right)}{\partial s_{2}}=\frac{1}{w_{3}-u_{3}} \int_{\gamma_{3}} \frac{\partial f\left(s_{1}, s_{2}, s_{3}\right)}{\partial s_{2}} d s_{3}+\frac{1}{w_{3}-u_{3}} \int_{\gamma_{3}} p_{3}\left(x_{3}, s_{3}\right) \frac{\partial^{2} f\left(s_{1}, s_{2}, s_{3}\right)}{\partial s_{3} \partial s_{2}} d s_{3} .
$$

Therefore we get

$$
\begin{array}{r}
A_{0}=\frac{1}{\prod_{i=1}^{3}\left(w_{i}-u_{i}\right)} \int_{\gamma_{1}} \int_{\gamma_{2}} \int_{\gamma_{3}} f\left(s_{1}, s_{2}, s_{3}\right) d s_{3} d s_{2} d s_{1}+\frac{1}{\prod_{i=1}^{3}\left(w_{i}-u_{i}\right)} \int_{\gamma_{1}} \int_{\gamma_{2}} \int_{\gamma_{3}} p_{3}\left(x_{3}, s_{3}\right) \frac{\partial f\left(s_{1}, s_{2}, s_{3}\right)}{\partial s_{3}} d s_{3} d s_{2} d s_{1} \\
+\frac{1}{\prod_{i=1}^{3}\left(w_{i}-u_{i}\right)} \int_{\gamma_{1}} \int_{\gamma_{2}} \int_{\gamma_{3}} p_{2}\left(x_{2}, s_{2}\right) \frac{\partial f\left(s_{1}, s_{2}, s_{3}\right)}{\partial s_{2}} d s_{3} d s_{2} d s_{1}+\frac{1}{\prod_{i=1}^{3}\left(w_{i}-u_{i}\right)} \int_{\gamma_{1}} \int_{\gamma_{2}} \int_{\gamma_{3}} p_{2}\left(x_{2}, s_{2}\right) p_{3}\left(x_{3}, s_{3}\right) \frac{\partial^{2} f\left(s_{1}, s_{2}, s_{3}\right)}{\partial s_{3} \partial s_{2}} d s_{3} d s_{2} d s_{1} .
\end{array}
$$

Similarly we obtain that

$$
\begin{aligned}
& B_{0}=\frac{1}{\prod_{i=1}^{3}\left(w_{i}-u_{i}\right)} \int_{\gamma_{1}} \int_{\gamma_{2}} \int_{\gamma_{3}} p_{1}\left(x_{1}, s_{1}\right) \frac{\partial f\left(s_{1}, s_{2}, s_{3}\right)}{\partial s_{1}} d s_{3} d s_{2} d s_{1}+ \\
& \frac{1}{\prod_{i=1}^{3}\left(w_{i}-u_{i}\right)} \int_{\gamma_{1}} \int_{\gamma_{2}} \int_{\gamma_{3}} p_{1}\left(x_{1}, s_{1}\right) p_{3}\left(x_{3}, s_{3}\right) \frac{\partial^{2} f\left(s_{1}, s_{2}, s_{3}\right)}{\partial s_{3} \partial s_{1}} d s_{3} d s_{2} d s_{1}+ \\
& \frac{1}{\prod_{i=1}^{3}\left(w_{i}-u_{i}\right)} \int_{\gamma_{1}} \int_{\gamma_{2}} \int_{\gamma_{3}} p_{1}\left(x_{1}, s_{1}\right) p_{2}\left(x_{2}, s_{2}\right) \frac{\partial^{2} f\left(s_{1}, s_{2}, s_{3}\right)}{\partial s_{2} \partial s_{1}} d s_{3} d s_{2} d s_{1}+ \\
& \frac{1}{\prod_{i=1}^{3}\left(w_{i}-u_{i}\right)} \int_{\gamma_{1}} \int_{\gamma_{2}} \int_{\gamma_{3}} p_{1}\left(x_{1}, s_{1}\right) p_{2}\left(x_{2}, s_{2}\right) p_{3}\left(x_{3}, s_{3}\right) \frac{\partial^{3} f\left(s_{1}, s_{2}, s_{3}\right)}{\partial s_{3} \partial s_{2} \partial s_{1}} d s_{3} d s_{2} d s_{1} .
\end{aligned}
$$

We have proved (2.1).

Next comes the general complex multivariate Montgomery type representation identity of complex functions:

Theorem 2.2. Let $f: \prod_{j=1}^{m} D_{j} \subseteq \mathbb{C}^{m} \rightarrow \mathbb{C}$ be a continuous function that is analytic per coordinate on the domain $D_{j}, j=1, \ldots, m$, and $x=\left(x_{1}, \ldots, x_{m}\right) \in \prod_{j=1}^{m} D_{j}$. For $j=1, \ldots, m$, suppose $\gamma_{j} \subset D_{j}$ is a smooth path parametrized by $z_{j}\left(t_{j}\right), t_{j} \in\left[a_{j}, b_{j}\right]$ with $z_{j}\left(a_{j}\right)=u_{j}, z_{j}\left(t_{j}\right)=x_{j}$ and $z_{j}\left(b_{j}\right)=w_{j}$, where $u_{j}, w_{j} \in D_{j}, u_{j} \neq w_{j}$. Assume also that all partial derivatives of $f$ up to order $m \in \mathbb{N}$ are continuous functions on $\prod_{j=1}^{m} D_{j}$.

We define the kernels $p_{i}: \gamma_{i}^{2} \rightarrow \mathbb{C}$

$$
p_{i}\left(x_{i}, s_{i}\right):= \begin{cases}s_{i}-u_{i}, & \text { if } s_{i} \in \gamma_{u_{i}, x_{i}}, \\ s_{i}-w_{i}, & \text { if } s_{i} \in \gamma_{x_{i}, w_{i}}\end{cases}
$$

for $i=1,2, \ldots, m$. 
Then

$$
\begin{aligned}
& f\left(x_{1}, x_{2}, \ldots, x_{m}\right)=\frac{1}{\prod_{i=1}^{m}\left(w_{i}-u_{i}\right)}\left\{\int_{\prod_{i=1}^{m} \gamma_{i}} f\left(s_{1}, s_{2}, \ldots, s_{m}\right) d s_{m} d s_{m-1} \ldots d s_{1}+\sum_{j=1}^{m}\left(\int_{\prod_{i=1}^{m} \gamma_{i}} p_{j}\left(x_{j}, s_{j}\right) \frac{\partial f\left(s_{1}, s_{2}, \ldots, s_{m}\right)}{\partial s_{j}} d s_{m} \ldots d s_{1}\right)+\right. \\
& \left(\sum_{\substack{l_{1}=1 \\
j<k}}^{\left(\begin{array}{c}
m \\
2
\end{array}\right)}\left(\int_{\prod_{i=1}^{m} \gamma_{i}} p_{j}\left(x_{j}, s_{j}\right) p_{k}\left(x_{k}, s_{k}\right) \frac{\partial^{2} f\left(s_{1}, s_{2}, \ldots, s_{m}\right)}{\partial s_{k} \partial s_{j}} d s_{m} \ldots d s_{1}\right)_{\left(l_{1}\right)}+\right. \\
& \left(\begin{array}{c}
m \\
3
\end{array}\right)\left(\int_{\substack{l_{2}=1 \\
j<k<r}} p_{j}\left(x_{j}, s_{j}\right) p_{k}\left(x_{k}, s_{k}\right) p_{r}\left(x_{r}, s_{r}\right) \frac{\partial^{3} f\left(s_{1}, \ldots, s_{m}\right)}{\partial s_{r} \partial s_{k} \partial s_{j}} d s_{m} \ldots d s_{1}\right)_{\left(l_{2}\right)}+\ldots+ \\
& \left.\left(\begin{array}{c}
m \\
m-1
\end{array}\right)\left(\int_{l=1}^{m} p_{1}\left(x_{1}, s_{1}\right) \ldots \widehat{p_{l}\left(x_{l}, s_{l}\right.}\right) \ldots p_{m}\left(x_{m}, s_{m}\right) \frac{\partial^{m-1} f\left(s_{1}, \ldots, s_{m}\right)}{\partial s_{m} \ldots \widehat{\partial s_{l} \ldots \partial s_{1}}} d s_{m} \ldots \widehat{d s_{l} \ldots d s_{1}}\right) \\
& \left.+\int \prod_{i=1}^{m} \gamma_{i}\left(\prod_{i=1}^{m} p_{i}\left(x_{i}, s_{i}\right)\right) \frac{\partial^{m} f\left(s_{1}, \ldots, s_{m}\right)}{\partial s_{m} \ldots \partial s_{1}} d s_{m} \ldots d s_{1}\right\} .
\end{aligned}
$$

Above $l_{1}$ counts $(j, k): j<k ; j, k \in\{1,2, \ldots, m\}$, also $l_{2}$ counts $(j, k, r): j<k<r ; j, k, r \in\{1,2, \ldots, m\}$, etc. Also $p_{l}\left(x_{l}, s_{l}\right)$ and $\widehat{\partial s_{l}}$ means that $p_{l}\left(x_{l}, s_{l}\right)$ and $\partial s_{l}$ are missing, respectively.

Proof. Similar to Theorem 2.1.

We make

Remark 2.3. (on Theorems 2.1, 2.2)

By (2.1) we get

$$
\begin{aligned}
& E_{f}\left(x_{1}, x_{2}, x_{3}\right):=f\left(x_{1}, x_{2}, x_{3}\right)-\frac{1}{\prod_{i=1}^{3}\left(w_{i}-u_{i}\right)}\left\{\int_{\prod_{i=1}^{3} \gamma_{i}} f\left(s_{1}, s_{2}, s_{3}\right) d s_{3} d s_{2} d s_{1}\right. \\
& \left.-\sum_{j=1}^{3}\left(\int_{i=1}^{3} \gamma_{i} p_{j}\left(x_{j}, s_{j}\right) \frac{\partial f\left(s_{1}, s_{2}, s_{3}\right)}{\partial s_{j}} d s_{3} d s_{2} d s_{1}\right)-\sum_{l=1}^{3}\left(\int_{j=k}^{3} \gamma_{i=1} p_{j}\left(x_{j}, s_{j}\right) p_{k}\left(x_{k}, s_{k}\right) \frac{\partial^{2} f\left(s_{1}, s_{2}, s_{3}\right)}{\partial s_{k} \partial s_{j}} d s_{3} d s_{2} d s_{1}\right)(l)\right\} \\
& =\frac{1}{\prod_{i=1}^{3}\left(w_{i}-u_{i}\right)}\left(\int_{\prod_{i=1}^{3} \gamma_{i}}\left(\prod_{i=1}^{3} p_{i}\left(x_{i}, s_{i}\right)\right) \frac{\partial^{3} f\left(s_{1}, s_{2}, s_{3}\right)}{\partial s_{3} \partial s_{2} \partial s_{1}} d s_{1} d s_{2} d s_{3}\right) .
\end{aligned}
$$

Above l counts $(j, k): j<k ; j, k \in\{1,2,3\}$.

Similarly, by (2.2) we find

$$
\begin{aligned}
& E_{f}\left(x_{1}, x_{2}, \ldots, x_{m}\right)=f\left(x_{1}, x_{2}, \ldots, x_{m}\right)- \\
& \frac{1}{\prod_{i=1}^{m}\left(w_{i}-u_{i}\right)}\left\{\int_{\prod_{i=1}^{m} \gamma_{i}} f\left(s_{1}, \ldots, s_{m}\right) d s_{1} \ldots d s_{m}-\sum_{j=1}^{m}\left(\int_{\prod_{i=1}^{m} \gamma_{i}} p_{j}\left(x_{j}, s_{j}\right) \frac{\partial f\left(s_{1}, \ldots, s_{m}\right)}{\partial s_{j}} d s_{1} \ldots d s_{m}\right)-\right.
\end{aligned}
$$




$$
\begin{aligned}
& \left(\sum_{\substack{l_{1}=1 \\
j<k}}^{\left(\begin{array}{c}
m \\
2
\end{array}\right)}\left(\int_{\prod_{i=1}^{m} \gamma_{i}} p_{j}\left(x_{j}, s_{j}\right) p_{k}\left(x_{k}, s_{k}\right) \frac{\partial^{2} f\left(s_{1}, \ldots, s_{m}\right)}{\partial s_{k} \partial s_{j}} d s_{1} \ldots d s_{m}\right)_{\left(l_{1}\right)}-\right. \\
& \sum_{\substack{l_{2}=1 \\
j<k<r}}^{\left(\begin{array}{c}
m \\
3
\end{array}\right)}\left(\int_{\prod_{i=1}^{m} \gamma_{i}} p_{j}\left(x_{j}, s_{j}\right) p_{k}\left(x_{k}, s_{k}\right) p_{r}\left(x_{r}, s_{r}\right) \frac{\partial^{3} f\left(s_{1}, \ldots, s_{m}\right)}{\partial s_{r} \partial s_{k} \partial s_{j}} d s_{1} \ldots d s_{m}\right)_{\left(l_{2}\right)}-\ldots- \\
& \left.\left(\begin{array}{c}
m \\
m-1
\end{array}\right)\left(\int_{l=1}^{m} p_{1}\left(x_{1}, s_{1}\right) \ldots \widehat{p_{l}\left(x_{l}, s_{l}\right)} \ldots p_{m}\left(x_{m}, s_{m}\right) \frac{\partial^{m-1} f\left(s_{1}, \ldots, s_{m}\right)}{\partial s_{m} \ldots \widehat{\partial s_{l}} \ldots \partial s_{1}} d s_{1} \ldots \widehat{d s_{l}} \ldots d s_{m}\right)\right\} \\
& =\frac{1}{\prod_{i=1}^{m}\left(w_{i}-u_{i}\right)}\left(\int_{\prod_{i=1}^{m} \gamma_{i}}\left(\prod_{i=1}^{m} p_{i}\left(x_{i}, s_{i}\right)\right) \frac{\partial^{m} f\left(s_{1}, \ldots, s_{m}\right)}{\partial s_{m} \ldots \partial s_{1}} d s_{1} \ldots d s_{m}\right) .
\end{aligned}
$$

Above $l_{1}$ counts $(j, k): j<k ; j, k \in\{1, \ldots, m\}, l_{2}$ counts $(j, k, r): j<k<r ; j, k, r \in\{1,2, \ldots, m\}$, etc. Also $\widehat{p_{l}\left(x_{l}, s_{l}\right)}$ and $\widehat{\partial s_{l}}$ means that $p_{l}\left(x_{l}, s_{l}\right)$ and $\partial s_{l}$ are missing, respectively.

Hence it holds

$$
\left|E_{f}\left(x_{1}, x_{2}, x_{3}\right)\right| \leq \frac{1}{\prod_{i=1}^{3}\left|w_{i}-u_{i}\right|} \times\left(\int_{\prod_{i=1}^{3} \gamma_{i}}\left(\prod_{i=1}^{3}\left|p_{i}\left(x_{i}, s_{i}\right)\right|\right)\left|\frac{\partial^{3} f\left(s_{1}, s_{2}, s_{3}\right)}{\partial s_{3} \partial s_{2} \partial s_{1}}\right|\left|d s_{1}\right|\left|d s_{2}\right|\left|d s_{3}\right|\right)
$$

and

$$
\left|E_{f}\left(x_{1}, \ldots, x_{m}\right)\right| \leq \frac{1}{\prod_{i=1}^{m}\left|w_{i}-u_{i}\right|} \times\left(\int_{\prod_{i=1}^{m} \gamma_{i}}\left(\prod_{i=1}^{m}\left|p_{i}\left(x_{i}, s_{i}\right)\right|\right)\left|\frac{\partial^{m} f\left(s_{1}, \ldots, s_{m}\right)}{\partial s_{m} \ldots \partial s_{1}}\right|\left|d s_{1}\right| \ldots\left|d s_{m}\right|\right) .
$$

We give the following complex multivariate Ostrowski type inequalities:

Theorem 2.4. All as in Theorem 2.1. Here $r_{1}, r_{2}, r_{3}, r_{4}>0: \frac{1}{r_{1}}+\frac{1}{r_{2}}+\frac{1}{r_{3}}+\frac{1}{r_{4}}=1$. Then

$$
\begin{aligned}
& \left|E_{f}\left(x_{1}, x_{2}, x_{3}\right)\right| \leq \frac{1}{\prod_{i=1}^{3}\left|w_{i}-u_{i}\right|} \times \min \left\{\left(\prod_{i=1}^{3} \int_{\gamma_{i}}\left|p_{i}\left(x_{i}, s_{i}\right)\right|\left|d s_{i}\right|\right)\left\|\frac{\partial^{3} f}{\partial s_{3} \partial s_{2} \partial s_{1}}\right\|_{\infty, \prod_{j=1}^{3} \gamma_{j}},\right. \\
& \left(\prod_{i=1}^{3}\left\|p_{i}\left(x_{i}, s_{i}\right)\right\|_{r_{j}, \gamma_{j}}\right)\left(\prod_{i=1}^{3}\left(l\left(\gamma_{j}\right)\right)^{\frac{2}{r_{j}}}\right)\left\|\frac{\partial^{3} f}{\partial s_{3} \partial s_{2} \partial s_{1}}\right\|_{r_{4}, \prod_{i=1}^{3} \gamma_{j}}, \\
& \left.\left(\prod_{\left(s_{1}, s_{2}, s_{3}\right) \in \prod_{j=1}^{3} \gamma_{j}}^{3}\left|p_{i=1}\left(x_{i}, s_{i}\right)\right|\right)\left\|\frac{\partial^{3} f}{\partial s_{3} \partial s_{2} \partial s_{1}}\right\| \prod_{j=1}^{3} \gamma_{j}\right\} \\
& \forall\left(x_{1}, x_{2}, x_{3}\right) \in \prod_{j=1}^{3} \gamma_{j} .
\end{aligned}
$$

Proof. By (2.3) and generalized Hölder's inequality. 
Theorem 2.5. All as in Theorem 2.2. Here $r_{1}, r_{2}, \ldots, r_{m}, r_{m+1}>0: \sum_{i=1}^{m+1} \frac{1}{r_{i}}=1$. Then

$$
\begin{aligned}
& \left|E_{f}\left(x_{1}, \ldots, x_{m}\right)\right| \leq \frac{1}{\prod_{i=1}^{m}\left|w_{i}-u_{i}\right|} \times \min \left\{\left(\prod_{i=1}^{m} \int_{\gamma_{i}}\left|p_{i}\left(x_{i}, s_{i}\right)\right|\left|d s_{i}\right|\right)\left\|\frac{\partial^{m} f}{\partial s_{m} \ldots \partial s_{1}}\right\|_{\infty, \prod_{j=1}^{m} \gamma_{j}},\right. \\
& \left(\prod_{i=1}^{m}\left\|p_{i}\left(x_{i}, s_{i}\right)\right\|_{r_{j}, \gamma_{j}}\right)\left(\prod_{i=1}^{m}\left(l\left(\gamma_{j}\right)\right)^{\frac{m-1}{r_{j}}}\right)\left\|\frac{\partial^{m} f}{\partial s_{m} \ldots \partial s_{1}}\right\|_{r_{m+1}, \prod_{i=1}^{m} \gamma_{j}}, \\
& \left(\begin{array}{l}
\left.\left.\sup _{\left(s_{1}, \ldots, s_{m}\right) \in \prod_{j=1}^{m} \gamma_{j}}\left(\prod_{i=1}^{m}\left|p_{i}\left(x_{i}, s_{i}\right)\right|\right)\right)\left\|\frac{\partial^{m} f}{\partial s_{m} \ldots \partial s_{1}}\right\|_{1, \prod_{j=1}^{m} \gamma_{j}}\right\} \\
\forall\left(x_{1}, \ldots, x_{m}\right) \in \prod_{j=1}^{m} \gamma_{j} .
\end{array}\right.
\end{aligned}
$$

Proof. By (2.4) and generalized Hölder's inequality.

We make

Remark 2.6. Working further on (2.1) we call:

$$
\begin{aligned}
& A_{f}^{(3)}:=A_{f}^{(3)}\left(x_{1}, x_{2}, x_{3}\right):=\sum_{j=1}^{3}\left(\int_{\prod_{i=1}^{3} \gamma_{i}} p_{j}\left(x_{j}, s_{j}\right) \frac{\partial f\left(s_{1}, s_{2}, s_{3}\right)}{\partial s_{j}} d s_{1} d s_{2} d s_{3}\right) \\
& +\sum_{\substack{l=1 \\
j<k}}^{3}\left(\int_{\prod_{i=1}^{3} \gamma_{i}} p_{j}\left(x_{j}, s_{j}\right) p_{k}\left(x_{k}, s_{k}\right) \frac{\partial^{2} f\left(s_{1}, s_{2}, s_{3}\right)}{\partial s_{k} \partial s_{j}} d s_{1} d s_{2} d s_{3}\right)(l),
\end{aligned}
$$

and

$$
B_{f}^{(3)}:=B_{f}^{(3)}\left(x_{1}, x_{2}, x_{3}\right):=\int_{\prod_{i=1}^{3} \gamma_{i}}\left(\prod_{i=1}^{3} p_{i}\left(x_{i}, s_{i}\right)\right) \frac{\partial^{3} f\left(s_{1}, s_{2}, s_{3}\right)}{\partial s_{3} \partial s_{2} \partial s_{1}} d s_{1} d s_{2} d s_{3} .
$$

Set also

$$
T_{f}^{(3)}:=T_{f}^{(3)}\left(x_{1}, x_{2}, x_{3}\right):=A_{f}^{(3)}+B_{f}^{(3)} .
$$

Thus, we have $\left(x=\left(x_{1}, x_{2}, x_{3}\right)\right)$

$$
\begin{aligned}
& f(x)=f\left(x_{1}, x_{2}, x_{3}\right)=\frac{1}{\prod_{i=1}^{3}\left(w_{i}-u_{i}\right)} \int_{\prod_{i=1}^{3} \gamma_{i}} f\left(s_{1}, s_{2}, s_{3}\right) d s_{1} d s_{2} d s_{3}+\frac{1}{\prod_{i=1}^{3}\left(w_{i}-u_{i}\right)}\left(A_{f}^{(3)}+B_{f}^{(3)}\right)= \\
& \frac{1}{\prod_{i=1}^{3}\left(w_{i}-u_{i}\right)} \int_{\prod_{i=1}^{3} \gamma_{i}} f\left(s_{1}, s_{2}, s_{3}\right) d s_{1} d s_{2} d s_{3}+\frac{1}{\prod_{i=1}^{3}\left(w_{i}-u_{i}\right)} T_{f}^{(3)} .
\end{aligned}
$$

Working further on (2.2) we call:

$$
A_{f}^{(m)}:=A_{f}^{(m)}\left(x_{1}, \ldots, x_{m}\right):=\sum_{j=1}^{m}\left(\int_{\prod_{i=1}^{m} \gamma_{i}} p_{j}\left(x_{j}, s_{j}\right) \frac{\partial f\left(s_{1}, \ldots, s_{m}\right)}{\partial s_{j}} d s_{1} \ldots d s_{m}\right)+
$$




$$
\begin{aligned}
& \left(\begin{array}{c}
m \\
2
\end{array}\right)\left(\int_{\substack{m \\
l_{1}=1 \\
j<k}} p_{j}\left(x_{j}, s_{j}\right) p_{k}\left(x_{k}, s_{k}\right) \frac{\partial^{2} f\left(s_{1}, \ldots, s_{m}\right)}{\partial s_{k} \partial s_{j}} d s_{1} \ldots d s_{m}\right)_{\left(l_{1}\right)}+ \\
& \left(\begin{array}{c}
m \\
3
\end{array}\right) \\
& \sum_{\substack{l_{2}=1 \\
j<k<r}}\left(\int_{\prod_{i=1}^{m} \gamma_{i}} p_{j}\left(x_{j}, s_{j}\right) p_{k}\left(x_{k}, s_{k}\right) p_{r}\left(x_{r}, s_{r}\right) \frac{\partial^{3} f\left(s_{1}, \ldots, s_{m}\right)}{\partial s_{r} \partial s_{k} \partial s_{j}} d s_{1} \ldots d s_{m}\right)\left(l_{2}\right)+\ldots+ \\
& \left.\left(\begin{array}{c}
m \\
m-1
\end{array}\right) \sum_{l=1}\left(\int_{\prod_{i=1}^{m} \gamma_{i}} p_{1}\left(x_{1}, s_{1}\right) \ldots p_{l} \widehat{\left(x_{l}, s_{l}\right.}\right) \ldots p_{m}\left(x_{m}, s_{m}\right) \frac{\partial^{m-1} f\left(s_{1}, \ldots, s_{m}\right)}{\partial s_{m} \ldots \widehat{\partial s_{l} \ldots \partial s_{1}}} d s_{1} \ldots \widehat{d s_{l} \ldots d s_{m}}\right)
\end{aligned}
$$

and

$$
B_{f}^{(m)}:=B_{f}^{(m)}\left(x_{1}, \ldots, x_{m}\right):=\int_{\prod_{i=1}^{m} \gamma_{i}}\left(\prod_{i=1}^{m} p_{i}\left(x_{i}, s_{i}\right)\right) \frac{\partial^{m} f\left(s_{1}, \ldots, s_{m}\right)}{\partial s_{m} \ldots \partial s_{1}} d s_{1} \ldots d s_{m} .
$$

Set also

$$
T_{f}^{(m)}:=T_{f}^{(m)}\left(x_{1}, \ldots, x_{m}\right):=A_{f}^{(m)}+B_{f}^{(m)}
$$

Thus, we have $\left(x=\left(x_{1}, \ldots, x_{m}\right)\right)$

$$
\begin{aligned}
& f(x)=f\left(x_{1}, \ldots, x_{m}\right)=\frac{1}{\prod_{i=1}^{m}\left(w_{i}-u_{i}\right)} \int_{\prod_{i=1}^{m} \gamma_{i}} f\left(s_{1}, \ldots, s_{m}\right) d s_{1} \ldots d s_{m}+\frac{1}{\prod_{i=1}^{m}\left(w_{i}-u_{i}\right)}\left(A_{f}^{(m)}+B_{f}^{(m)}\right)= \\
& \frac{1}{\prod_{i=1}^{m}\left(w_{i}-u_{i}\right)} \int_{\prod_{i=1}^{m} \gamma_{i}} f\left(s_{1}, \ldots, s_{m}\right) d s_{1} \ldots d s_{m}+\frac{1}{\prod_{i=1}^{m}\left(w_{i}-u_{i}\right)} T_{f}^{(m)} .
\end{aligned}
$$

Let function $g$ as in Theorem 2.2. Then as in (2.5) we obtain

$$
\begin{aligned}
& g(x)=g\left(x_{1}, \ldots, x_{m}\right)=\frac{1}{\prod_{i=1}^{m}\left(w_{i}-u_{i}\right)} \int_{\prod_{i=1}^{m} \gamma_{i}} g\left(s_{1}, \ldots, s_{m}\right) d s_{1} \ldots d s_{m}+\frac{1}{\prod_{i=1}^{m}\left(w_{i}-u_{i}\right)}\left(A_{g}^{(m)}+B_{g}^{(m)}\right)= \\
& \frac{1}{\prod_{i=1}^{m}\left(w_{i}-u_{i}\right)} \int_{\prod_{i=1}^{m} \gamma_{i}} g\left(s_{1}, \ldots, s_{m}\right) d s_{1} \ldots d s_{m}+\frac{1}{\prod_{i=1}^{m}\left(w_{i}-u_{i}\right)} T_{g}^{(m)} .
\end{aligned}
$$

Above $A_{g}^{(m)}, B_{g}^{(m)}, T_{g}^{(m)}$ have the obvious meaning.

By (2.5) we get

$$
f(x) g(x)=\frac{g(x)}{\prod_{i=1}^{m}\left(w_{i}-u_{i}\right)} \int_{\prod_{i=1}^{m} \gamma_{i}} f\left(s_{1}, \ldots, s_{m}\right) \prod_{i=1}^{m} d s_{i}+\frac{g(x)}{\prod_{i=1}^{m}\left(w_{i}-u_{i}\right)} T_{f}^{(m)},
$$

and by (2.6) we get

$$
g(x) f(x)=\frac{f(x)}{\prod_{i=1}^{m}\left(w_{i}-u_{i}\right)} \int_{\prod_{i=1}^{m} \gamma_{i}} g\left(s_{1}, \ldots, s_{m}\right) \prod_{i=1}^{m} d s_{i}+\frac{f(x)}{\prod_{i=1}^{m}\left(w_{i}-u_{i}\right)} T_{g}^{(m)} .
$$

Consequently after integration we get: 
$\left(\right.$ set $\left.s:=\left(s_{1}, \ldots, s_{m}\right)\right)$

$$
\int_{\prod_{i=1}^{m} \gamma_{i}} f(s) g(s) \prod_{i=1}^{m} d s_{i}=\frac{\int_{i=1}^{m} \gamma_{i} g(s) \prod_{i=1}^{m} d s_{i}}{\prod_{i=1}^{m}\left(w_{i}-u_{i}\right)} \int_{\prod_{i=1}^{m} \gamma_{i}} f(s) \prod_{i=1}^{m} d s_{i}+\frac{1}{\prod_{i=1}^{m}\left(w_{i}-u_{i}\right)} \int_{\prod_{i=1}^{m} \gamma_{i}} g(s) T_{f}^{(m)}(s) \prod_{i=1}^{m} d s_{i},
$$

and

$$
\int_{\prod_{i=1}^{m} \gamma_{i}} f(s) g(s) \prod_{i=1}^{m} d s_{i}=\frac{\int_{i=1}^{m} \gamma_{i} f(s) \prod_{i=1}^{m} d s_{i}}{\prod_{i=1}^{m}\left(w_{i}-u_{i}\right)} \int_{\prod_{i=1}^{m} \gamma_{i}} g(s) \prod_{i=1}^{m} d s_{i}+\frac{1}{\prod_{i=1}^{m}\left(w_{i}-u_{i}\right)} \int_{\prod_{i=1}^{m} \gamma_{i}} f(s) T_{g}^{(m)}(s) \prod_{i=1}^{m} d s_{i} .
$$

By (2.7) and (2.8) we obtain

$$
\begin{aligned}
& \int_{i=1}^{m} \gamma_{i} f(s) g(s) \prod_{i=1}^{m} d s_{i}-\frac{1}{\prod_{i=1}^{m}\left(w_{i}-u_{i}\right)}\left(\int_{\prod_{i=1}^{m} \gamma_{i}} f(s) \prod_{i=1}^{m} d s_{i}\right)\left(\int_{\prod_{i=1}^{m} \gamma_{i}} g(s) \prod_{i=1}^{m} d s_{i}\right)=\frac{1}{\prod_{i=1}^{m}\left(w_{i}-u_{i}\right)} \int_{\prod_{i=1}^{m} \gamma_{i}} f(s) T_{g}^{(m)}(s) \prod_{i=1}^{m} d s_{i}= \\
& \quad \frac{1}{\prod_{i=1}^{m}\left(w_{i}-u_{i}\right)} \int_{\prod_{i=1}^{m} \gamma_{i}} g(s) T_{f}^{(m)}(s) \prod_{i=1}^{m} d s_{i} .
\end{aligned}
$$

We conclude that (set $d \vec{s}:=\prod_{i=1}^{m} d s_{i}$ )

$\int_{i=1}^{m} \gamma_{i} f(s) g(s) d \vec{s}-\frac{1}{\prod_{i=1}^{m}\left(w_{i}-u_{i}\right)}\left(\int_{\prod_{i=1}^{m} \gamma_{i}} f(s) d \vec{s}\right)\left(\int_{\prod_{i=1}^{m} \gamma_{i}} g(s) d \vec{s}\right)=\frac{1}{2\left(\prod_{i=1}^{m}\left(w_{i}-u_{i}\right)\right)}\left[\int_{\prod_{i=1}^{m} \gamma_{i}}\left(f(s) T_{g}^{(m)}(s)+g(s) T_{f}^{(m)}(s)\right) d \vec{s}\right]$.

Therefore we have

$$
\begin{aligned}
& \frac{1}{\prod_{i=1}^{m}\left(w_{i}-u_{i}\right)} \int_{\prod_{i=1}^{m} \gamma_{i}} f(s) g(s) d \vec{s}-\frac{1}{\prod_{i=1}^{m}\left(w_{i}-u_{i}\right)}\left(\int_{\prod_{i=1}^{m} \gamma_{i}} f(s) d \vec{s}\right) \frac{1}{\prod_{i=1}^{m}\left(w_{i}-u_{i}\right)}\left(\int_{\prod_{i=1}^{m} \gamma_{i}} g(s) d \vec{s}\right)=\frac{1}{2\left(\prod_{i=1}^{m}\left(w_{i}-u_{i}\right)\right)^{2}} \\
& {\left[\int_{i=1}^{m}\left\{f(s)\left(A_{g}^{(m)}(s)+B_{g}^{(m)}(s)\right)+g(s)\left(A_{f}^{(m)}(s)+B_{f}^{(m)}(s)\right)\right\} d \vec{s}\right] .}
\end{aligned}
$$

Hence it holds

$$
\begin{array}{r}
\Delta(f, g):=\frac{1}{\prod_{i=1}^{m}\left(w_{i}-u_{i}\right)} \int_{\prod_{i=1}^{m} \gamma_{i}} f(s) g(s) d \vec{s}-\frac{1}{\prod_{i=1}^{m}\left(w_{i}-u_{i}\right)}\left(\int_{\prod_{i=1}^{m} \gamma_{i}} f(s) d \vec{s}\right) \frac{1}{\prod_{i=1}^{m}\left(w_{i}-u_{i}\right)}\left(\int_{\prod_{i=1}^{m} \gamma_{i}} g(s) d \vec{s}\right)- \\
\frac{1}{2\left(\prod_{i=1}^{m}\left(w_{i}-u_{i}\right)\right)^{2}}\left[\int_{\prod_{i=1}^{m} \gamma_{i}}\left\{f(s) A_{g}^{(m)}(s)+g(s) A_{f}^{(m)}(s)\right\} d \vec{s}\right]=\frac{1}{2\left(\prod_{i=1}^{m}\left(w_{i}-u_{i}\right)\right)^{2}}\left[\int_{\prod_{i=1}^{m} \gamma_{i}}\left\{f(s) B_{g}^{(m)}(s)+g(s) B_{f}^{(m)}(s)\right\} d \vec{s}\right] .
\end{array}
$$

Clearly we derive that $\left(|d \vec{s}|:=\prod_{i=1}^{m}\left|d s_{i}\right|\right)$

$$
|\Delta(f, g)| \leq \frac{1}{2\left(\prod_{i=1}^{m}\left|w_{i}-u_{i}\right|\right)^{2}}\left[\int_{i=1}^{m}\left\{|f(s)|\left|B_{g}^{(m)}(s)\right|+|g(s)|\left|B_{f}^{(m)}(s)\right|\right\}|d \vec{s}|\right]=
$$




$$
\begin{aligned}
& \frac{1}{2\left(\prod_{i=1}^{m}\left|w_{i}-u_{i}\right|\right)^{2}}\left[\int_{\prod_{i=1}^{m} \gamma_{i}}|f(s)|\left|B_{g}^{(m)}(s)\right||d \vec{s}|+\int_{\prod_{i=1}^{m} \gamma_{i}}|g(s)|\left|B_{f}^{(m)}(s)\right||d \vec{s}|\right] \leq \\
& \frac{1}{2\left(\prod_{i=1}^{m}\left|w_{i}-u_{i}\right|\right)^{2}}\left[\|f\|_{\infty, \prod_{i=1}^{m} \gamma_{i}}\left(\int_{\prod_{i=1}^{m} \gamma_{i}}\left|B_{g}^{(m)}(s)\right||d \vec{s}|\right)+\|g\|_{\infty, \prod_{i=1}^{m} \gamma_{i}}\left(\int_{\prod_{i=1}^{m} \gamma_{i}}\left|B_{f}^{(m)}(s)\right||d \vec{s}|\right)\right] .
\end{aligned}
$$

We have established the following complex multivariate Grüss type inequality:

Theorem 2.7. Let $f, g$ and all as in Theorem 2.2. Then

$$
\begin{aligned}
& \mid \frac{1}{\prod_{i=1}^{m}\left(w_{i}-u_{i}\right)} \int_{\prod_{i=1}^{m} \gamma_{i}} f(s) g(s) d \vec{s}-\frac{1}{\prod_{i=1}^{m}\left(w_{i}-u_{i}\right)}\left(\int_{\prod_{i=1}^{m} \gamma_{i}} f(s) d \vec{s}\right) \frac{1}{\prod_{i=1}^{m}\left(w_{i}-u_{i}\right)}\left(\int_{\prod_{i=1}^{m} \gamma_{i}} g(s) d \vec{s}_{i}\right)- \\
& \frac{1}{2\left(\prod_{i=1}^{m}\left(w_{i}-u_{i}\right)\right)^{2}}\left[\int_{\prod_{i=1}^{m} \gamma_{i}}\left(f(s) A_{g}^{(m)}(s)+g(s) A_{f}^{(m)}(s)\right) d \vec{s}\right] \mid \leq \\
& \frac{1}{2\left(\prod_{i=1}^{m}\left|w_{i}-u_{i}\right|\right)^{2}}\left[\|f\|_{\infty} \prod_{i=1}^{m} \gamma_{i}\right. \\
&
\end{aligned}
$$

The corresponding $L_{p}$ Grüss inequality follows:

Theorem 2.8. Let $f, g$ and all as in Theorem 2.2 and $p, q>1$ such that $\frac{1}{p}+\frac{1}{q}=1$. Then

$$
\begin{aligned}
& \mid \frac{1}{\prod_{i=1}^{m}\left(w_{i}-u_{i}\right)} \int_{i=1}^{m} f(s) g(s) d \vec{s}-\frac{1}{\prod_{i=1}^{m}\left(w_{i}-u_{i}\right)}\left(\int_{\prod_{i=1}^{m} \gamma_{i}} f(s) d \vec{s}\right) \frac{1}{\prod_{i=1}^{m}\left(w_{i}-u_{i}\right)}\left(\int_{\prod_{i=1}^{m} \gamma_{i}} g(s) d \vec{s}\right)- \\
& \frac{1}{2\left(\prod_{i=1}^{m}\left(w_{i}-u_{i}\right)\right)^{2}}\left[\int_{\prod_{i=1}^{m} \gamma_{i}}\left(f(s) A_{g}^{(m)}(s)+g(s) A_{f}^{(m)}(s)\right) d \vec{s}\right] \mid \leq \\
& \frac{1}{2\left(\prod_{i=1}^{m}\left|w_{i}-u_{i}\right|\right)^{2}}\left[\|f\|_{p, \prod_{i=1}^{m} \gamma_{i}}\left\|B_{g}^{(m)}\right\|_{q, \prod_{i=1}^{m} \gamma_{i}}+\|g\|_{p, \prod_{i=1}^{m} \gamma_{i}}\left\|B_{f}^{(m)}\right\|_{q, \prod_{i=1}^{m} \gamma_{i}}\right]
\end{aligned}
$$

Proof. Use of (2.9) and Hölder inequality.

The corrsponding $L_{1}$ Grüss inequality follows:

Theorem 2.9. Let $f, g$ and all as in Theorem 2.2. Then

$$
\mid \frac{1}{\prod_{i=1}^{m}\left(w_{i}-u_{i}\right)} \int_{\prod_{i=1}^{m} \gamma_{i}} f(s) g(s) d \vec{s}-\frac{1}{\prod_{i=1}^{m}\left(w_{i}-u_{i}\right)}\left(\int_{\prod_{i=1}^{m} \gamma_{i}} f(s) d \vec{s}\right) \frac{1}{\prod_{i=1}^{m}\left(w_{i}-u_{i}\right)}\left(\int_{\prod_{i=1}^{m} \gamma_{i}} g(s) d \vec{s}\right)-
$$




$$
\begin{aligned}
& \frac{1}{2\left(\prod_{i=1}^{m}\left(w_{i}-u_{i}\right)\right)^{2}}\left[\int_{\prod_{i=1}^{m} \gamma_{i}}\left(f(s) A_{g}^{(m)}(s)+g(s) A_{f}^{(m)}(s)\right) d \vec{s}\right] \mid \leq \\
& \frac{1}{2\left(\prod_{i=1}^{m}\left|w_{i}-u_{i}\right|\right)^{2}}\left[\|f\|_{1, \prod_{i=1}^{m} \gamma_{i}}\left\|B_{g}^{(m)}\right\|_{\infty, \prod_{i=1}^{m} \gamma_{i}}+\|g\|_{1, \prod_{i=1}^{m} \gamma_{i}}\left\|B_{f}^{(m)}\right\|_{\infty, \prod_{i=1}^{m} \gamma_{i}}\right] .
\end{aligned}
$$

Proof. By (2.9)

Corollary 2.10. Let $f, g$ and all as in Theorem 2.1. Then

$$
\begin{aligned}
& \mid \frac{1}{\prod_{i=1}^{3}\left(w_{i}-u_{i}\right)} \int_{i=1}^{3} \gamma_{i} f(s) g(s) d \vec{s}-\frac{1}{\prod_{i=1}^{3}\left(w_{i}-u_{i}\right)}\left(\int_{\prod_{i=1}^{3} \gamma_{i}} f(s) d \vec{s}\right) \frac{1}{\prod_{i=1}^{3}\left(w_{i}-u_{i}\right)}\left(\int_{\prod_{i=1}^{3} \gamma_{i}} g(s) d \vec{s}\right)- \\
& \frac{1}{2\left(\prod_{i=1}^{3}\left(w_{i}-u_{i}\right)\right)^{2}}\left[\int_{\prod_{i=1}^{3} \gamma_{i}}\left(f(s) A_{g}^{(3)}(s)+g(s) A_{f}^{(3)}(s)\right) d \vec{s}\right] \mid \leq \\
& \frac{1}{2\left(\prod_{i=1}^{m}\left|w_{i}-u_{i}\right|\right)^{2}}\left[\|f\|_{\infty, \prod_{i=1}^{3} \gamma_{i}}\left(\int_{\prod_{i=1}^{3} \gamma_{i}}\left|B_{g}^{(3)}(s)\right||d \vec{s}|\right)+\|g\| \|_{\infty, \prod_{i=1}^{3} \gamma_{i}}\left(\int_{\prod_{i=1}^{3} \gamma_{i}}\left|B_{f}^{(3)}(s)\right||d \vec{s}|\right)\right] .
\end{aligned}
$$

Proof. By Theorem 2.7 for $m=3$.

Corollary 2.11. Let $f, g$ and all as in Theorem 2.1 and $p, q>1: \frac{1}{p}+\frac{1}{q}=1$. Then

$$
\begin{aligned}
& \mid \frac{1}{\prod_{i=1}^{3}\left(w_{i}-u_{i}\right)} \int_{\prod_{i=1}^{3} \gamma_{i}} f(s) g(s) d \vec{s}-\frac{1}{\prod_{i=1}^{3}\left(w_{i}-u_{i}\right)}\left(\int_{\prod_{i=1}^{3} \gamma_{i}} f(s) d \vec{s}\right) \frac{1}{\prod_{i=1}^{3}\left(w_{i}-u_{i}\right)}\left(\int_{\prod_{i=1}^{3} \gamma_{i}} g(s) d \vec{s}\right)- \\
& \frac{1}{2\left(\prod_{i=1}^{3}\left(w_{i}-u_{i}\right)\right)^{2}}\left[\int_{\prod_{i=1}^{3} \gamma_{i}}\left(f(s) A_{g}^{(3)}(s)+g(s) A_{f}^{(3)}(s)\right) d \vec{s}\right] \mid \leq \\
& \frac{1}{2\left(\prod_{i=1}^{3}\left|w_{i}-u_{i}\right|\right)^{2}}\left[\|f\|_{p, \prod_{i=1}^{3} \gamma_{i}}\left\|B_{g}^{(3)}\right\|_{q, \prod_{i=1}^{3} \gamma_{i}}+\|g\|_{p, \prod_{i=1}^{3} \gamma_{i}}\left\|B_{f}^{(3)}\right\|_{q, \prod_{i=1}^{3} \gamma_{i}}\right]
\end{aligned}
$$

Proof. By Theorem 2.8 for $m=3$.

Corollary 2.12. Let $f, g$ and all as in Theorem 2.1. Then

$$
\mid \frac{1}{\prod_{i=1}^{3}\left(w_{i}-u_{i}\right)} \int_{\prod_{i=1}^{3} \gamma_{i}} f(s) g(s) d \vec{s}-\frac{1}{\prod_{i=1}^{3}\left(w_{i}-u_{i}\right)}\left(\int_{\prod_{i=1}^{3} \gamma_{i}} f(s) d \vec{s}\right) \frac{1}{\prod_{i=1}^{3}\left(w_{i}-u_{i}\right)}\left(\int_{\prod_{i=1}^{3} \gamma_{i}} g(s) d \vec{s}\right)-
$$




$$
\begin{aligned}
& \frac{1}{2\left(\prod_{i=1}^{3}\left(w_{i}-u_{i}\right)\right)^{2}}\left[\int_{\prod_{i=1}^{3} \gamma_{i}}\left(f(s) A_{g}^{(3)}(s)+g(s) A_{f}^{(3)}(s)\right) d \vec{s}\right] \mid \leq \\
& \frac{1}{2\left(\prod_{i=1}^{3}\left|w_{i}-u_{i}\right|\right)^{2}}\left[\|f\|_{1, \prod_{i=1}^{3} \gamma_{i}}\left\|B_{g}^{(3)}\right\|_{\infty, \prod_{i=1}^{3} \gamma_{i}}+\|g\|_{1, \prod_{i=1}^{3} \gamma_{i}}\left\|B_{f}^{(3)}\right\|_{\infty, \prod_{i=1}^{3} \gamma_{i}}\right] .
\end{aligned}
$$

Proof. By Theorem 2.9 for $m=3$.

\section{References}

[1] A. Ostrowski, Über die Absolutabweichung einer differentiebaren Funktion von ihrem Integral mittelwert, Comment. Math. Helv. 10 (1938), 226-227.

[2] G. Grüss, Über das Maximum des absoluten Betrages von $\frac{1}{b-a} \int_{a}^{b} f(x) g(x) d x-\frac{1}{(b-a)^{2}} \int_{a}^{b} f(x) d x \int_{a}^{b} g(x) d x$, Math. Z., 39 (1935), 215-226.

[3] D. S. Mitrinović, J.E. Pečarić, A.M. Fink, Inequalities for Functions and Their Integrals and Derivatives, Kluwer Academic Publishers, Dordrecht, 1994.

[4] S. S. Dragomir, An extension of Ostrowski's inequality to the Complex integral, RGMIA Res. Rep. Call. 21 (2018), Art. 112, $17 \mathrm{pp}$, [http://rgmia.org/papers/v21/v21/v21a112.pdf].

[5] S. S. Dragomir, On some Grüss type inequalities for the complex integral, RGMIA Res. Rep. Call. 21 (2018), Art. 121, 12 pp, [http://rgmia.org/papers/v21/v21a121.pdf]. 